\title{
Lytic bacteriophages against multidrug-resistant Staphylococcus aureus, Enterococcus faecalis and Escherichia coli isolates from orthopaedic implant-associated infections
}

\author{
Joana Barros a,b,c,*, Luís D.R. Melo ${ }^{\mathrm{d}}$, Patrícia Poeta ${ }^{\mathrm{e}, \mathrm{f}}$, Gilberto Igrejass ${ }^{\mathrm{f} g, \mathrm{~h}}$, Maria P. Ferraz ${ }^{\mathrm{i}}$, \\ Joana Azeredo ${ }^{\mathrm{d}}$, Fernando J. Monteiro ${ }^{\mathrm{a}, \mathrm{b}, \mathrm{c}}$ \\ a i3S-Instituto de Investigação e Inovação em Saúde, Universidade do Porto, Rua Alfredo Allen 208, 4200-135 Porto, Portugal \\ b INEB-Instituto Nacional de Engenharia Biomédica, Porto, Portugal \\ ${ }^{\mathrm{c}}$ FEUP-Faculdade de Engenharia, Universidade do Porto, Porto, Portugal \\ ${ }^{\mathrm{d}}$ Laboratório de Investigação em Biofilmes Rosário Oliveira, Centre of Biological Engineering, University of Minho, Braga, Portugal \\ e Department of Veterinary Sciences, University of Trás-os-Montes and Alto Douro, Vila Real, Portugal \\ ${ }^{\mathrm{f}}$ LAQV-REQUIMTE, Faculty of Science and Technology, Nova University of Lisbon, Lisbon, Portugal \\ ${ }^{g}$ Department of Genetics and Biotechnology, University of Trás-os-Montes and Alto Douro, Vila Real, Portugal \\ ${ }^{\mathrm{h}}$ Functional Genomics and Proteomics Unit, University of Trás-os-Montes and Alto Douro, Vila Real, Portugal

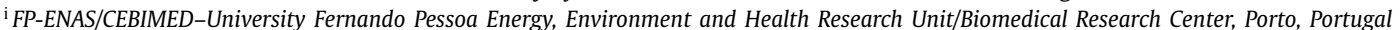

\section{A R T I C L E I N F O}

\section{Article history:}

Received 22 August 2018

Accepted 10 June 2019

Editor: Prof. Geoffrey Coombs

Keywords:

Implant-associated infection

Pathogenic bacteria

Multidrug resistance

Phage therapy

Efficiency

Specificity

\begin{abstract}
A B S T R A C T
Orthopaedic implant-associated infections are a devastating complication of orthopaedic surgery with a significant impact on patients and healthcare systems. The aims of this work were to describe the patterns of antimicrobial resistance, pathogenicity and virulence of clinical bacterial isolates from orthopaedic implant-associated infections and to further isolate and characterise bacteriophages that are efficient in controlling these bacteria. Staphylococcus aureus, Enterococcus faecalis and Escherichia coli isolated from orthopaedic infections showed multiresistance patterns to the most frequently used antibiotics in clinical settings. The presence of mobile genetic elements (mecA, Tn916/Tn1545 and intl1) and virulence determinants (icaB, cna, hlb, cylLs, cylM, agg, gelE, fsr and fimA) highlighted the pathogenicity of these isolates. Moreover, the isolates belonged to clonal complexes associated with the acquisition of pathogenicity islands and antimicrobial resistance genes by recombination and horizontal gene transfer. Bacteriophages vB_SauM_LM12, vB_EfaS_LM99 and vB_EcoM_JB75 were characterised and their ability to infect clinical isolates of $S$. aureus, E. faecalis and E. coli, respectively, was assessed. Morphological and genomic analyses revealed that vB_EfaS_LM99 and vB_EcoM_JB75 belong to the Siphoviridae and Myoviridae families, respectively, and no genes associated with lysogeny were found. The bacteriophages showed low latent periods, high burst sizes, broad host ranges and tolerance to several environmental conditions. Moreover, they showed high efficiency and specificity to infect and reduce clinical bacteria, including methicillinresistant $S$. aureus and vancomycin-resistant enterococci. Therefore, the results obtained suggest that the bacteriophages used in this work are a promising approach to control these pathogens involved in orthopaedic implant-associated infections.
\end{abstract}

(c) 2019 Elsevier B.V. and International Society of Chemotherapy. All rights reserved.

\section{Introduction}

Orthopaedic implant-associated infections are a devastating complication of orthopaedic surgery with a significant impact on patient quality of life and healthcare systems [1]. The most commonly isolated bacteria from these infections are Staphylococ-

\footnotetext{
* Corresponding author. Tel.: +351220408800.

E-mail addresses: joana.barros@ineb.up.pt, joana_rbarros@hotmail.com (J. Barros).
}

cus aureus (33-43\%), Staphylococcus epidermidis (18-40\%) and Enterococcus spp. (2.5-15\%, mainly Enterococcus faecalis). However, Gram-negative bacilli, including Escherichia coli and Pseudomonas aeruginosa, are less frequent causes of implant-associated infection $(4-7 \%)[2-4]$.

The emergence of antimicrobial-resistant bacteria and their ability to produce virulence factors have contributed to enhancing the pathogenicity and severity of orthopaedic implantassociated infections [3,4-7]. According to the World Health Organization (WHO), these bacteria, namely methicillin-resistant 
Table 1

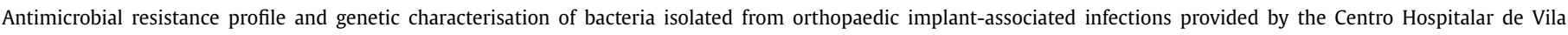
Nova de Gaia/Espinho, EPE (Vila Nova de Gaia, Portugal).

\begin{tabular}{|c|c|c|c|c|c|c|c|c|}
\hline Species & Strain & Source & Patient sex/age & Antimicrobial resistance ${ }^{a}$ & \multicolumn{4}{|c|}{ Genetic characterisation } \\
\hline Enterobacter cloacae & 2107408 & Thigh bone & $\mathrm{F} / 76$ & STR/CIP/FOX/CEF/AMX/SUL/MFX/TMP & & & & \\
\hline Klebsiella pneumoniae & 2133201 & Shoulder & $\mathrm{M} / 39$ & AMX/NEO & & & & \\
\hline Acinetobacter lwoffii & 2170401 & Hip prosthesis & $\mathrm{M} / 74$ & FEP & & & & \\
\hline \multirow{2}{*}{ Pseudomonas aeruginosa } & 209960 & Lumbar arthrodesis & $\mathrm{F} / 69$ & ATM/GEN/NET & & & & \\
\hline & & & & & Type & VFs & ST & $\mathrm{CC}$ \\
\hline Staphylococcus & 2117045 & Synovial fluid & $\mathrm{F} / 72$ & ERY/FOX/CIP/GEN/AMP & MRSA & $i c a B-c n a-h l b$ & 239 & 8 \\
\hline \multirow{6}{*}{ aureus } & 2093367 & Knee & $\mathrm{F} / 60$ & ERY/FOX/CIP/AMP & MRSA & cna & 22 & 22 \\
\hline & 2104780 & Ankle & $\mathrm{F} / 75$ & ERY/FOX/CIP/AMP & MRSA & $i c a B-c n a-h l b$ & 22 & 22 \\
\hline & 2117741 & Knee & $\mathrm{M} / 70$ & ERY/FOX/CIP/GEN/AMP & MRSA & $i c a B-c n a-h l b$ & 22 & 22 \\
\hline & 2106876 & Hip prosthesis & $\mathrm{F} / 53$ & GEN/AMP & MSSA & $i c a B-h l b$ & 72 & 8 \\
\hline & 2179342 & Synovial fluid & $\mathrm{M} / 42$ & GEN/AMK/AMP & MSSA & $i c a B-c n a-h l b$ & 72 & 8 \\
\hline & & & & & Transposon & VFs & ST & $\mathrm{CC}$ \\
\hline Enterococcus & 2099610 & Lumbar arthrodesis & $\mathrm{F} / 69$ & QDA/CIP/VAN & $\mathrm{N} / \mathrm{D}$ & cylLs & 117 & 21 \\
\hline \multirow{7}{*}{ faecalis } & 2105322 & Septic arthritis & $\mathrm{M} / 77$ & ERY/QDA/CIP/VAN & $\mathrm{N} / \mathrm{D}$ & agg-gelE-fsr-cylLs & 117 & 21 \\
\hline & 2104780 & Ankle & $\mathrm{F} / 75$ & ERY/QDA/CIP/TET/NOR/VAN/TEC & $\operatorname{Tn} 916 / \operatorname{Tn} 1545$ & agg-fsr-cylLs & 6 & 2 \\
\hline & 2133201 & Shoulder & $\mathrm{M} / 39$ & ERY/QDA/CIP/TET/NOR/VAN/TEC & $\operatorname{Tn} 916 / \operatorname{Tn} 1545$ & agg-cylM-fsr-cylLs & 16 & 58 \\
\hline & 2084972 & Soft foot bones & $\mathrm{F} / 79$ & ERY/QDA/CIP/TET/NOR/VAN/TEC & $\operatorname{Tn} 916 / \operatorname{Tn} 1545$ & agg-cylLs & 6 & 2 \\
\hline & 2093926 & Knee & $\mathrm{M} / 76$ & ERY/QDA/CIP/VAN & $\mathrm{N} / \mathrm{D}$ & $a g g-f s r-c y l L s$ & 117 & 21 \\
\hline & 8105329 & Knee prosthesis & $\mathrm{M} / 75$ & ERY/QDA/VAN & $\mathrm{N} / \mathrm{D}$ & agg-gelE-fsr-cylLs & 117 & 21 \\
\hline & & & & & Phylogenetic group & VFs & \multicolumn{2}{|c|}{ Integron } \\
\hline \multirow{2}{*}{$\begin{array}{l}\text { Escherichia } \\
\text { coli }\end{array}$} & 2129975 & Iliac crest & $\mathrm{M} / 86$ & CIP/CEF/MFX/TMP/NEO/NAL/OFX & B1 & fimA & \multicolumn{2}{|c|}{ intl1 } \\
\hline & 2154120 & Hip prosthesis & $\mathrm{M} / 47$ & CEF/TMP/NEO/NAL/OFX & A & fimA & \multicolumn{2}{|l|}{$\mathrm{N} / \mathrm{D}$} \\
\hline
\end{tabular}

VF, virulence factor; ST, sequence type; CC, clonal complex; MRSA, methicillin-resistant S. aureus; MSSA, methicillin-susceptible S. aureus; N/D, not detected.

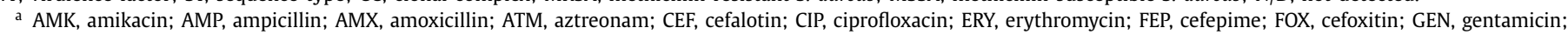

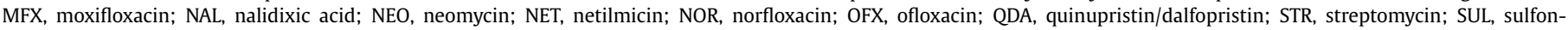
amides; TEC, teicoplanin; TET, tetracycline; TMP, trimethoprim; VAN, vancomycin.

S. aureus (MRSA) and vancomycin-resistant enterococci (VRE), are classified as a serious threat to public health owing to limited therapeutic options [8].

To address this situation, the use of bacteriophages (phages) has been extensively studied as an alternative therapeutic strategy [9]. Phages are bacterial viruses that specifically infect bacteria, hijacking their machinery, replicating intracellularly and finally lysing the host bacterium $[7,10]$. In vitro and in vivo studies have shown that for several situations, phage therapy is more specific, accurate and without adverse effects/local tissue toxicity compared with antibiotic therapy $[7,10,11]$. Characteristics such as bioavailability at the site of infection and migratory ability to other infection sites make this therapy more attractive.

Therefore, phage therapy could be a promising alternative strategy to treat orthopaedic implant-associated infections. The purposes of this work were primarily to describe the patterns of antimicrobial resistance, pathogenicity and virulence of bacteria isolated from orthopaedic implant-associated infections and further to isolate and characterise phages that are efficient in controlling these pathogens.

\section{Materials and methods}

\subsection{Characterisation of bacterial isolates}

Bacterial isolates related to orthopaedic implant-associated infections were provided by Centro Hospitalar de Vila Nova de Gaia/Espinho, EPE (CHVNG) (Vila Nova de Gaia, Portugal) (Table 1). Over a 5-month period, 18 samples from osteoarticular infections were collected from patients at CHVNG. Biological samples were collected enabling the isolation and identification of 19 bacterial isolates using VITEK $® 2$ Compact Bacterial Identification and Monitoring System (bioMérieux Inc., Durham, NC) following standardised protocols implemented at CHVNG. The study was approved by the hospital ethics committee, and patient records were anonymised prior to analysis.

\subsubsection{Antimicrobial resistance and genomic characterisation}

Clinical isolates were subjected to antimicrobial susceptibility testing by the disk diffusion method according to European Committee on Antimicrobial Susceptibility Testing (EUCAST) and Clinical and Laboratory Standards Institute (CLSI) guidelines [12,13]. The antimicrobial classes, antibiotic concentration and zone diameter breakpoint for each bacterial species were defined according to EUCAST and CLSI recommendations following the disk diffusion antimicrobial susceptibility testing method.

Genomic DNA of each isolate was extracted using specific methods: for S. aureus, lysostaphin/proteinase K/Tris-HCl [14]; for E. faecalis, InstaGene ${ }^{\mathrm{TM}}$ matrix [15]; and for E. coli, boiling [15].

MRSA identification was performed by amplification of the mecA and nucC genes [16]. Detection of tndX and int genes in E. faecalis isolates was performed to demonstrate the presence of Tn5397-like and Tn916/Tn1545-like transposons, respectively [15]. The intI 1 and intl2 genes, encoding class 1 and 2 integrases, respectively, were amplified in E. coli isolates [15].

For $E$. coli, the phylogenetic group (A, B1, B2 and D) was identified by amplification of the chuA and $y j a A$ genes as well as DNA fragment TspE4.C2 [15,17,18].

Screening of virulence genes for S. aureus (cna, eta, etb, tst, $h l b, i c a A, i c a B$ and $i c a C)$, E. faecalis (ace, agg, gelE, esp, hyl, fsr and $\operatorname{cylL}_{L} L_{S} A B M$ ) and E. coli (fimA, papGIII, stx, cnf1 and papC) was performed by PCR [14-18].

Positive and negative controls were used from the bacterial collection of the Medical Microbiology Laboratory of the Universidade de Trás-os-Montes e Alto Douro (UTAD) (Vila Real, Portugal). A list of the primers used is provided in the Supplementary material SS1.

\subsubsection{Multilocus sequence typing (MLST)}

Staphylococcus aureus and E. faecalis isolates were characterised by MLST. Internal fragments of seven housekeeping genes of $S$. aureus (arcC, aroE, glpF, gmk, pta, tpi and yqiL) and E. faecalis (gdh, gyd, pstS, gki, aroE, xpt and yiqL) were amplified using the primers listed in Supplementary material SS1 and were sequenced. The obtained sequences were analysed by https://pubmlst.org/general.shtml and eBURST V3 to assign a specific sequence type (ST) and clonal com- 
plex (CC). Positive and negative controls were used from the bacterial collection of the Medical Microbiology Laboratory at UTAD.

\subsection{Bacteriophage isolation/production and characterisation}

A previously isolated phage from the Bacteriophage Biotechnology Group of the Centre for Biological Engineering (BBiG/CEBUniversidade do Minho, Braga, Portugal) was used to infect $S$. aureus isolates [19]. Specific new phages towards E. faecalis and E. coli strains were isolated from a wastewater treatment plant at Frossos (Braga, Portugal). A sample enrichment method was performed to isolate phages [20]. Briefly, centrifuged effluent was mixed with double-strength trypticase soy broth and exponentially grown $E$. faecalis and E. coli strains, respectively. The solution was then incubated at $37{ }^{\circ} \mathrm{C}$ and $120 \mathrm{rpm}$ for $24 \mathrm{~h}$ and was further centrifuged and the supernatant was filtered through a PES $0.22 \mu \mathrm{M}$ filter. Spot assays were performed against bacterial lawns to test for the presence of phages. Inhibition haloes were further purified and plaque picking was repeated until single-plaque morphology was observed.

Phages were produced as previously described with some modifications [21]. Briefly, phage solutions were spread on lawns of their respective host strains (S. aureus 12, E. faecalis 99 and $E$. coli 2129975) using a paper strip and were incubated overnight at $37{ }^{\circ} \mathrm{C}$. After full lysis, salt magnesium buffer was added to each plate and the plates were incubated at $4{ }^{\circ} \mathrm{C}$ and $120 \mathrm{rpm}$ for 24 h. Subsequently, both liquid and top agar were collected and centrifuged and the supernatant was filtered. Chloroform was added to the filtered solution and the samples were stored at $4{ }^{\circ} \mathrm{C}$ for further use.

Lytic spectra and efficiency of plating (EOP) were determined according to Kvachadze et al. [22]. In brief, phage suspensions were serially diluted and were placed over original or target host bacteria and the presence of a clear zone of lysis was examined following incubation at $37{ }^{\circ} \mathrm{C}$ for $16-18 \mathrm{~h}$,. The relative EOP was calculated as the ratio of the phage titre (PFU/mL) obtained in each isolate and that obtained in the propagating host. Three independent experiments were performed in duplicate.

\subsubsection{Thermal and $\mathrm{pH}$ stability tests}

To assess thermostability, phage solutions were incubated at different temperatures $\left(-20^{\circ} \mathrm{C}\right.$ to $\left.60{ }^{\circ} \mathrm{C}\right)$ for $24 \mathrm{~h}$. To assess pH stability, phage suspensions were prepared at different $\mathrm{pH}$ values $(\mathrm{pH}$ 1-13) and were incubated at $4{ }^{\circ} \mathrm{C}$ for $24 \mathrm{~h}$. In both cases, following incubation phages were titrated using the double-layer agar plate method to determine surviving phages. Three independent experiments were performed in duplicate.

\subsubsection{Bacteriophage morphology}

Phages were sedimented by centrifugation and the pellet was washed in tap water by repeating the centrifugation step [23]. Phages were deposited on copper grids with a carbon-coated Formvar film grid, were stained with $2 \%$ uranyl acetate $(\mathrm{pH} 4)$ and were examined using a JEOL JEM transmission electron microscope (JEOL, Tokyo, Japan).

\subsubsection{One-step growth curves}

One-step growth curves were performed as previously described [21]. Briefly, host bacteria were grown to exponential phase and were then harvested and re-suspended in fresh medium. Respective phage solutions were added to exponential-phase cultures of host bacteria at a multiplicity of infection (MOI) of 0.01 and were allowed to adsorb for $5 \mathrm{~min}$ at $37^{\circ} \mathrm{C}$. The mixtures were centrifuged and the pellets were then re-suspended in fresh medium broth. Samples were taken at 10-min intervals and phage titration was performed by the double-layer agar plating method. Three independent experiments were performed in duplicate.

\subsubsection{Genome sequencing analysis}

Escherichia phage vB_EcoM_JB75 (JB75) and Enterococcus phage vB_EfaS_LM99 (LM99) genomic DNA was extracted essentially as previously described [24]. In brief, purified phages were treated with $0.016 \%(\mathrm{v} / \mathrm{v}) \mathrm{L} 1$ buffer at $37{ }^{\circ} \mathrm{C}$ for $2 \mathrm{~h}$. The enzymes were further thermally inactivated for $30 \mathrm{~min}$ at $65{ }^{\circ} \mathrm{C}$. Then, phage proteins were digested with $50 \mu \mathrm{g} / \mathrm{mL}$ proteinase $\mathrm{K}, 20 \mathrm{mM}$ ethylene diamine tetra-acetic acid (EDTA) and 1\% sodium dodecyl sulfate (SDS) at $56{ }^{\circ} \mathrm{C}$ for $18 \mathrm{~h}$. This was followed by phenol, phenol:chloroform $(1: 1, \mathrm{v} / \mathrm{v})$ and chloroform extractions. DNA was precipitated with ice-cold absolute ethanol and $3 \mathrm{M}$ sodium acetate ( $\mathrm{pH}$ 4.6) and was then centrifuged. Pellets were washed in $70 \%$ ice-cold ethanol and were further air-dried and re-suspended in nuclease-free water. Phage genomes were sequenced using an Illumina HiSeq system (Illumina Inc., San Diego, CA) with individual libraries of two non-homologous phages pooled together in equal amounts. Libraries were constructed using the KAPA DNA Library Preparation Kit Illumina (KAPA Biosystems, San Diego, CA) with the KAPA HiFi preparation protocol and were sequenced using 100-bp paired-end mode. The quality of the produced data was determined by Phred quality score at each cycle. Reads were demultiplexed and de novo assembled into a single contig with average coverage above $100 \times$ using CLC Genomics Workbench v.7.0 (CLC Bio, Aarhus, Denmark) and were manually inspected.

Phage genomes were first annotated using myRAST algorithm and were further manually inspected for potential alternative start codons or for the presence of non-annotated coding sequences (CDSs) using Geneious 9.1.4 (Biomatters Ltd., Auckland, New Zealand). Functions of the gene products were searched with BLASTp (coverage $>80 \%$; E-value $\leq 10^{-5}$ ) and Pfam programs $\left(E\right.$-value $\left.\leq 10^{-5}\right)$. The presence of transmembrane domains was checked using TMHMM and Phobius, and membrane proteins were annotated when both tools were in concordance. Protein parameters (molecular weight and isoelectric point) were determined using ExPASy Compute $\mathrm{pl} / \mathrm{Mw}$ tool. Moreover, transfer RNAs (tRNAs) were scanned using tRNAscan-SE and ARAGORN. Promoter regions were determined using PromoterHunter from the phiSITE database and were further checked manually. ARNold was used to predict rho-independent terminators, and the energy was calculated using Mfold.

\subsection{Activity of bacteriophages against clinical bacteria}

The activity of phages against the bacterial isolates from orthopaedic implant-associated infections was evaluated. Bacterial cultures were grown to exponential phase and were re-suspended in fresh medium. Phage solutions were added to bacterial cultures of each respective bacterium at a MOI of 10 . These suspensions were incubated at $37{ }^{\circ} \mathrm{C}$ at $120 \mathrm{rpm}$ for 2,6 and $24 \mathrm{~h}$. The number of cultivable cells was determined using the microdrop method. Three independent experiments were performed in duplicate.

\subsection{Statistical analysis}

Experimental data were analysed using IBM SPSS Statistics v.22.0 (IBM Corp., Armonk, NY). Results were reported as the mean \pm standard deviation. One-way analysis of variance (ANOVA) followed by post-hoc Turkey HSD multiple comparison test was used to determine significant differences $(P<0.05)$.

\section{Results}

\subsection{Antimicrobial resistance and genomic characterisation}

A total of 19 clinical bacteria were isolated from orthopaedic implant-associated infections, showing resistance to several 
antibiotics (Table 1). Among these, 15 isolates were resistant to at least one antibiotic in three or more antimicrobial classes (Table 1), thus showing a multidrug-resistant (MDR) profile [25]. For further analysis, only isolates with a MDR profile were taken into consideration for further genomic characterisation as well as isolation and characterisation of phages against these target bacteria. This option was based on the therapeutic limitations for treating infections caused by MDR bacteria, with phage therapy being a possible solution.

Among six $S$. aureus isolates, four were MRSA and two isolates were methicillin-susceptible S. aureus (MSSA) (Table 1). All S. aureus isolates were shown to contain virulence determinants including polysaccharide intercellular adhesion gene (icaB), collagenbinding adhesin gene ( $\mathrm{cna}$ ) and haemolytic toxin $\beta$-haemolysin gene $(h l b)$ (Table 1$)$.

All seven E. faecalis isolates were vancomycin-resistant, three of which were also resistant to teicoplanin. The Tn916/Tn1545 transposon was found in the latter isolates. Moreover, cytolysins (cylLs and cylM), aggregation protein (agg), gelatinase (gelE) and pheromone gelatinase biosynthesis-activating pheromone $(f s r)$ virulence genes were found in the E. faecalis isolates (Table 1).

Lastly, the two E. coli isolates were classified into phylogenetic groups A and B1. The type 1 fimbriae gene (fimA) was detected in both isolates. Remarkably, the E. coli isolate classified into phylogenetic group B1 possessed the class 1 integron gene intl1 (Table 1).

\subsubsection{Multilocus sequence typing}

The $S$. aureus isolates were divided into three STs, namely ST239, ST72 and ST22 (Table 1). The MRSA isolates belonged to ST239 and ST22, whilst the MSSA isolates belonged to ST72. According to eBURST V3 analysis, $S$. aureus isolates belonged to CC8 (ST239 and ST72) and CC22 (ST22), (Table 1; Supplementary Fig. S1). Isolates assigned to ST239 and ST72 (Table 1) belonged to the same cluster, having closely related genotypes (Supplementary Fig. S1). CC8 had a primary founder ST5, whilst CC22 had as the primary founder ST22.

Regarding E. faecalis, the isolates were divided into three different STs belonging to three CCs: ST117 belonging to CC21; ST6 belonging to CC2; and ST16 belonging to CC58 (Table 1; Supplementary Fig. S1). CC21 had a primary founder ST21, whilst CC2 and CC58 had as the primary founders ST6 and ST16, respectively.

\subsection{Bacteriophage isolation/production and characterisation}

The phages used in this study were named vB_SauM_LM12 (LM12), vB_EfaS_LM99 (LM99), vB_EfaS_LM00I (LM00I), vB_EfaS_LM00II (LM00II), vB_EcoM_JB75 (JB75) and vB_EcoM_JB75I (JB75I) according to the recommendations of Kropinski et al. [26]. One phage (LM12) was used to infect S. aureus isolates (Table 2), whilst three phages (LM99, LM00I and LM00II) and two phages (JB75 and JB75I) were isolated to infect E. faecalis and E. coli isolates, respectively (Table 2). Phages LM12, LM99 and JB75 were able to lyse $91 \%, 64 \%$ and $55 \%$ of all S. aureus, E. faecalis and E. coli tested, respectively (Table 2). These phages were selected for further characterisation owing to their broad spectra of activity.

The EOP was determined in bacterial isolates from orthopaedic implant-associated infections (Table 2). Phage LM12 was able to infect all $S$. aureus isolates, with a high EOP in five of six $S$. aureus isolates (Table 2). Phage LM99 was able to infect one E. faecalis isolate with high lytic efficiency and to promote lysis from without in another two isolates (Table 2). Furthermore, phage JB75 infected only one E. coli isolate with high EOP.

\subsubsection{Thermal and $\mathrm{pH}$ stability tests}

The thermal stability of phage LM12 was assessed and a $100 \%$ survival rate was observed at $4{ }^{\circ} \mathrm{C}$ and $18{ }^{\circ} \mathrm{C}$, whilst at $37{ }^{\circ} \mathrm{C}$
Table 2

Lytic spectrum and efficiency of plating (EOP) of phages against Staphylococcus aureus, Enterococcus faecalis and Escherichia coli strains.

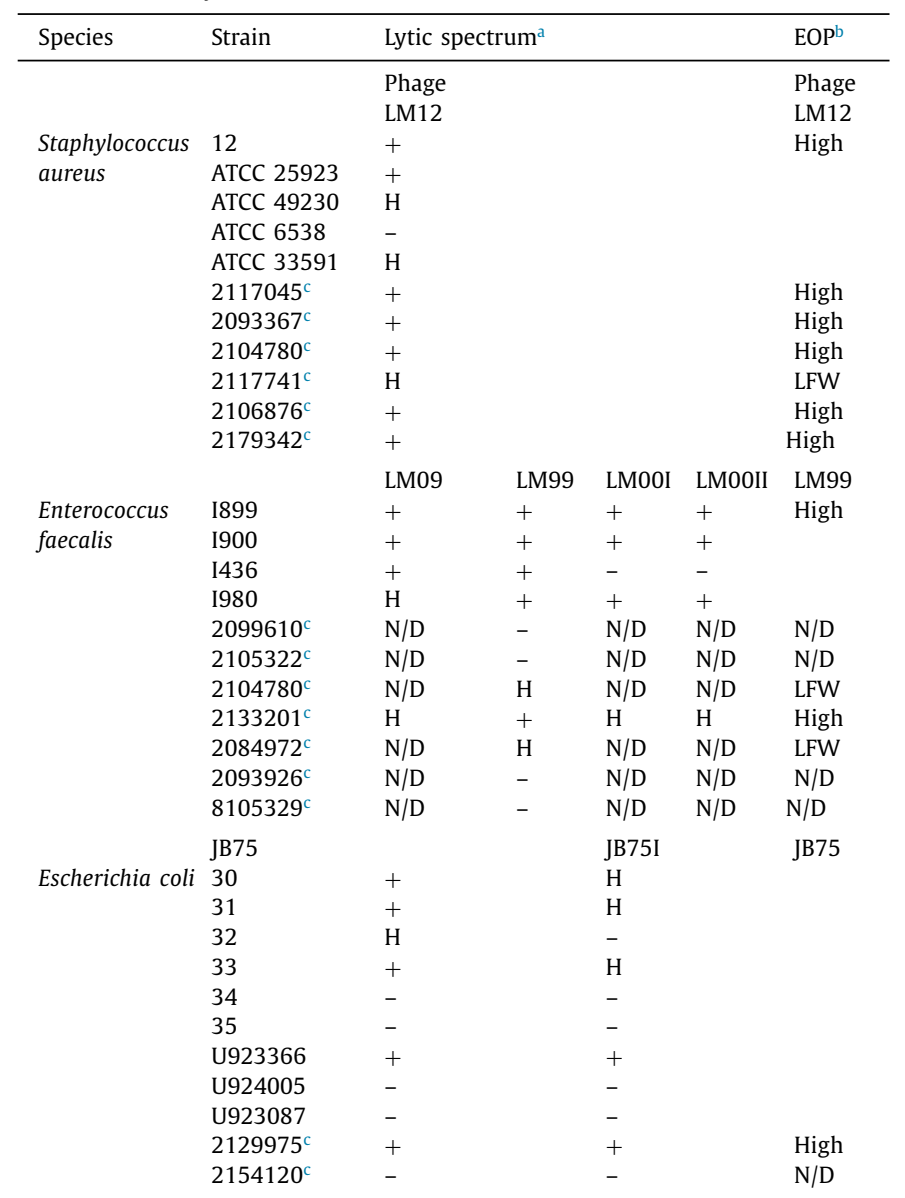

LFW, lysis from without; N/D, not determined.

a + , distinct clear plaques; -, plaques not formed; $\mathrm{H}$, hazy plaques.

b The EOP was recorded as high, low and LFW, representing $>10,0.1-1$ and $<0.1 \%$, respectively.

${ }^{\mathrm{c}}$ Bacteria isolated from orthopaedic implant-associated infections.

and $42{ }^{\circ} \mathrm{C}$ the survival rates were $98 \%$ and $94 \%$, respectively (Fig. 1a). This phage was able to survive at $-20{ }^{\circ} \mathrm{C}$ ( $42 \%$ survival rate), whereas it was killed at $60{ }^{\circ} \mathrm{C}$ (Fig. 1a). Regarding the thermal stability of phage LM99, a 100\% survival rate was observed for a wide range of temperatures from $-20{ }^{\circ} \mathrm{C}$ to $42{ }^{\circ} \mathrm{C}$ (Fig. 1a). Furthermore, the survival rate at $60{ }^{\circ} \mathrm{C}$ was approximately $39 \%$. Phage JB75 was shown to be more sensitive to temperature, being stable from $4-42{ }^{\circ} \mathrm{C}$. The survival rate was $75 \%$ at $-20{ }^{\circ} \mathrm{C}$, whilst no survival was detected at $60{ }^{\circ} \mathrm{C}$ (Fig. 1a).

Regarding $\mathrm{pH}$ stability, the three phages were highly stable over a wide range of $\mathrm{pH}$ values (Fig. 1b). Phage LM12 showed a loss of stability at $\mathrm{pH} 10$ (70\% survival rate), whilst the stability of phage LM99 was affected at $\mathrm{pH} 4$ and $\mathrm{pH} 12$ (78\% and 67\% survival rates, respectively) (Fig. 1b). The phages were completely inactivated at extreme $\mathrm{pH}$ values ( $\mathrm{pH} \mathrm{1,2}$ and 13) (Fig. 1b).

\subsubsection{Phage morphology}

Phage LM99 particles had an icosahedral head of $63 \mathrm{~nm}$ in diameter and a non-contractile tail of $212 \mathrm{~nm}$ in length and $9 \mathrm{~nm}$ in width (Fig. 1c), proving that it belongs to the Siphoviridae family. Phage JB75 revealed an isometric head of $73 \mathrm{~nm}$ diameter with a contractile tail $96 \mathrm{~nm}$ long and $24 \mathrm{~nm}$ wide, which is a morphology indicative of the Myoviridae family (Fig. 1c). 

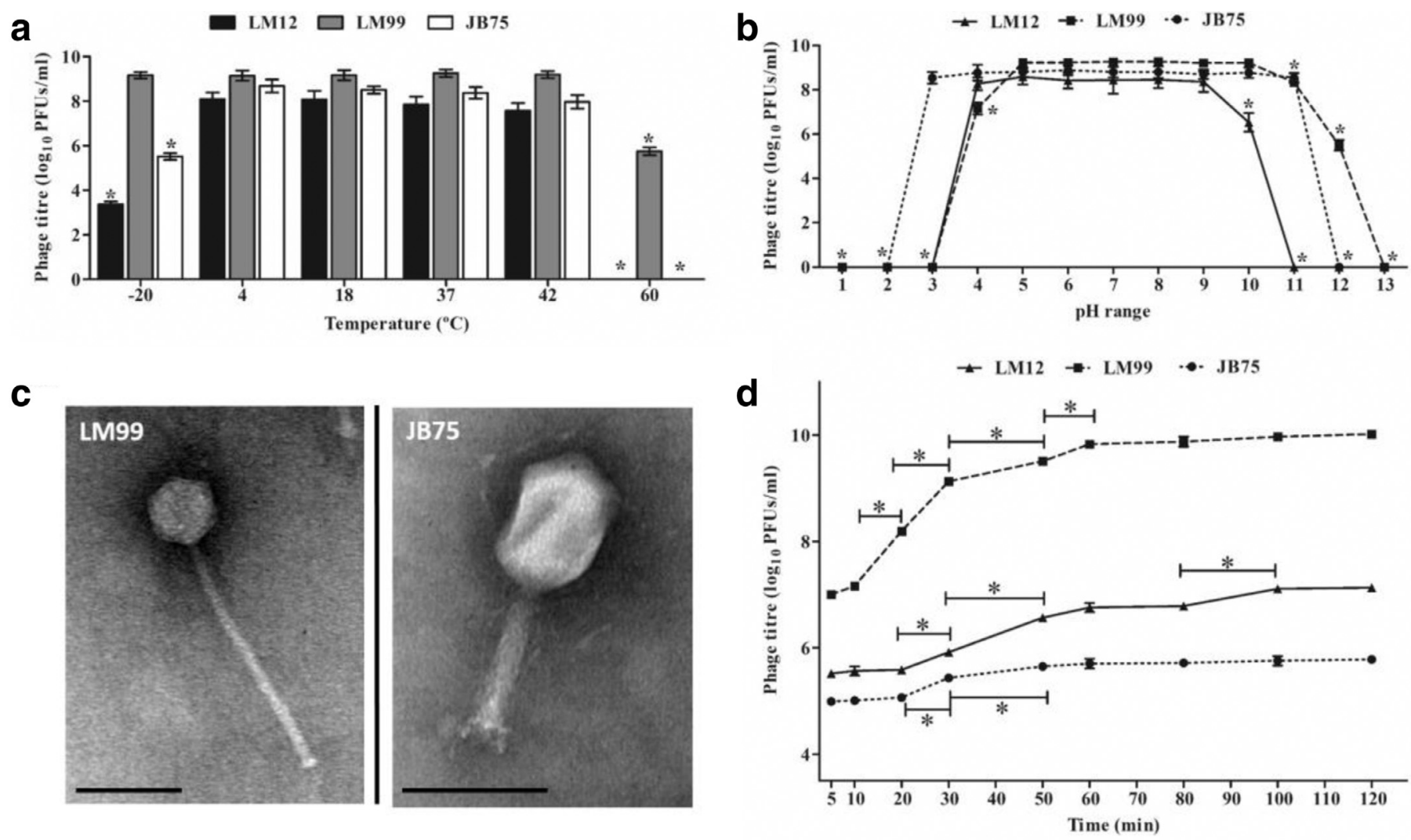

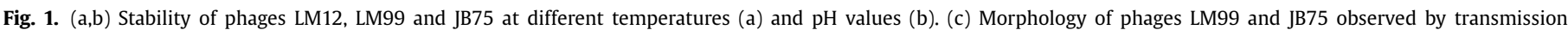

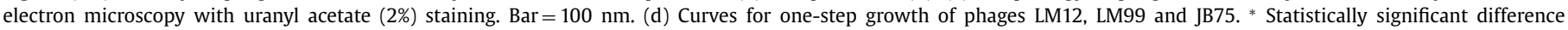
$(P<0.05)$.

\subsubsection{One-step growth curves}

The latent and rise periods for phage LM12 were $20 \mathrm{~min}$ and 30 min, respectively. The burst size was 52 PFU/infected cell (Fig. 1d). Regarding phage LM99, the latent and rise periods were $10 \mathrm{~min}$ and $20 \mathrm{~min}$, respectively, and the burst size was $107 \mathrm{PFU} /$ infected cell (Fig. 1d). Regarding phage JB75, the latent and rise periods were $20 \mathrm{~min}$ and $30 \mathrm{~min}$, respectively, and the burst size was 82 PFU/infected cell (Fig. 1d).

\subsubsection{Genomic sequencing analysis}

The complete genomic sequences of phages LM99 and JB75 were deposited in GenBank under the accession nos. MH355583 and MH355584, respectively. Genome analysis revealed that both phages are virulent, not encoding any genes associated with lysogeny. However, phage LM99 encodes a gene homologous to a putative toxin gene (gp17 - haemolysin) and a metallo- $\beta$ lactamase gene. In silico analysis showed that phage JB75 does not encode any known virulence-associated or toxin proteins.

The genome of phage LM99 consists of a linear double-stranded DNA of 40203 bp with a G+C\% content of 30.5\% (Fig. 2a). LM99 encodes 64 CDSs with an average length of $573 \mathrm{bp}$, tightly packed occupying $91 \%$ of its genome. Twenty-five of the predicted CDSs have an assigned function (39\%) and two are unique (Supplementary Table S1). No tRNA genes were detected. The majority (97\%) of the CDSs possess methionine as start codon, whilst CTG and GTG are the start codons of only one CDS each. Furthermore, 14 promoters and 6 rho-independent terminators were predicted. BLASTN search revealed high homology with enterococci siphoviruses vB_EfaS_AL3, LY0322, SHEF5, SHEF2, PMBT2, SANTOR1 and EfaCPT1. Interestingly, these phages are not inserted in any genus.
The genome of phage JB75 consists of a linear double-stranded DNA of 167208 bp with a G+C\% content of 35\% (Fig. 2b). This phage encodes 277 putative CDSs with an average length of 546 bp, also very tightly packed occupying approximately $94 \%$ of its genome. No unique proteins were detected and it was possible to predict a function for 134 CDSs (48\%) (Supplementary Table S2). Unsurprisingly, 262 CDSs have methionine as start codon (95\%), whilst 2 start with CTG, 6 with GTG and 7 with TTG. Ten tRNA genes were predicted (tRNA-Arg, tRNA-Asn, tRNA-Gln, tRNAGly, tRNA-Leu, tRNA-Met, tRNA-Pro, tRNA-Ser, tRNA-Thr and tRNATyr). Moreover, 13 promoters and 26 rho-independent terminators were predicted. Homology searches revealed that JB75 has very high homologies with several E. coli myoviruses, namely YUEEL01, vB_EcoM-fHoEco02 and vB_EcoM-fFiEco06. These phages are inserted in the T4 virus genus.

\subsection{Activity of bacteriophages against clinical bacteria}

The antimicrobial activity of the phages was assessed against bacterial isolates with high EOP values (Table 2). In the control group (without phage), S. aureus, E. faecalis and E. coli bacterial counts increased continuously (Fig. 3, solid lines). However, when phages LM12, LM99 and JB75 were applied, significant reductions were observed in the tested isolates (Fig. 3, dotted lines). Noticeably, the phage effect varied according to the bacterial strain tested.

Phage LM12 showed high antimicrobial activity for the four $S$. aureus isolates tested (Fig. 3a). Despite the slight increase in bacterial counts observed at $6 \mathrm{~h}$ and $24 \mathrm{~h}$, bacterial counts were significantly lower compared with the controls $(P<0.05)$. Phage LM12 reduced the bacterial density of $S$. aureus 2093367 by $91 \%, 97 \%$ and $99 \%$ at 2, 6 and 24 h, respectively. Reductions of approximately 


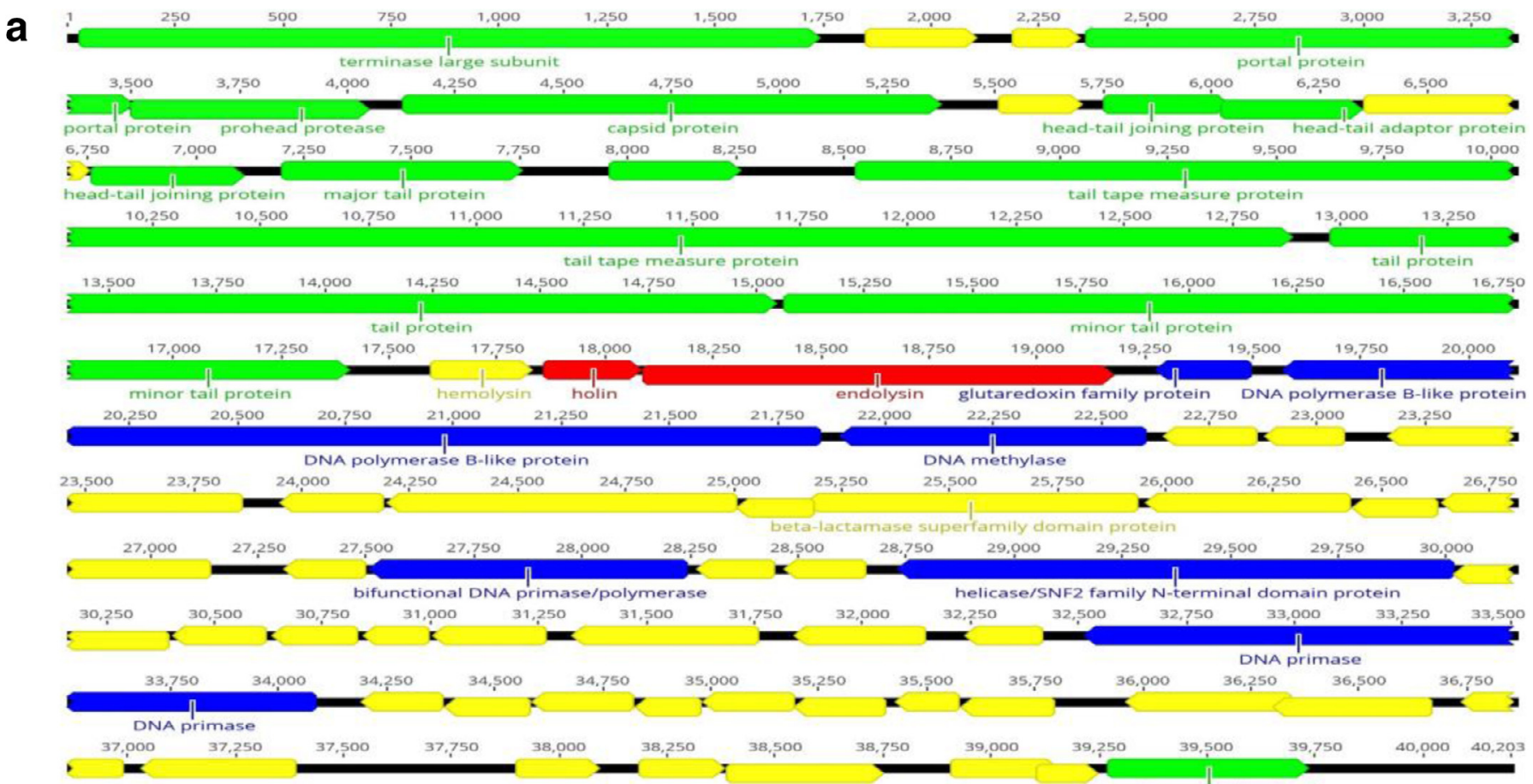

terminase small subunit

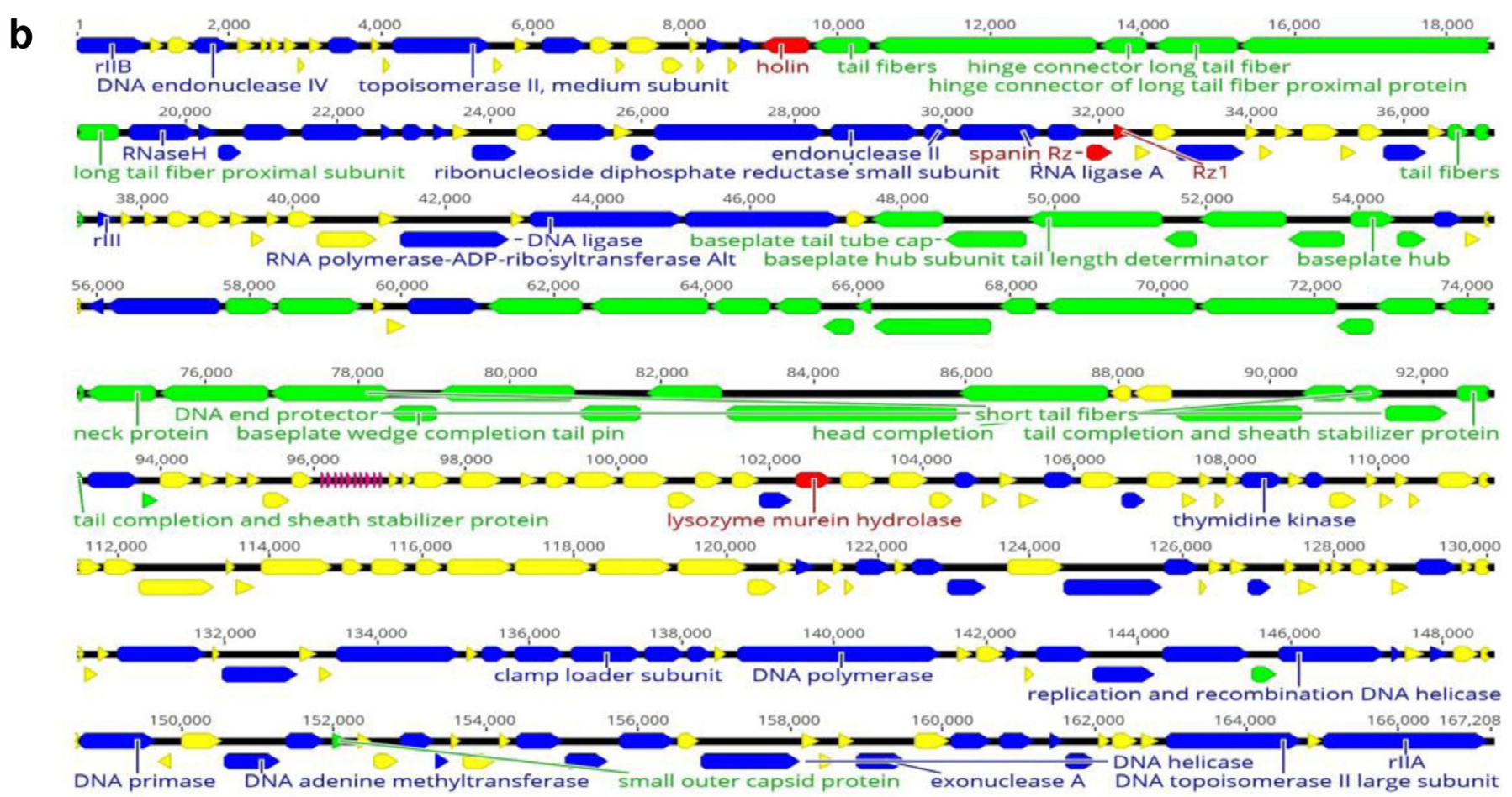

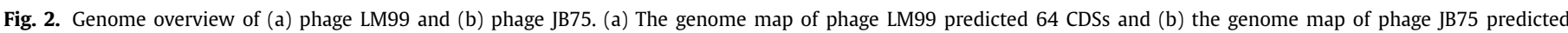

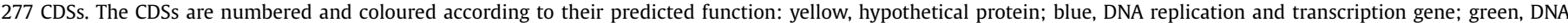

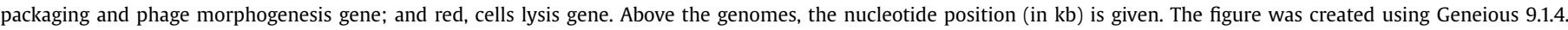
CDS, coding sequence.

96\%, 95\% and 93\% at 2, 6 and 24 h, respectively, were observed in S. aureus 2104780, S. aureus 2106876 and S. aureus (Fig. 3a). Regarding S. aureus 2117045 and S. aureus 2117741, the antimicrobial effect of LM12 decreased over time, showing a maximum effect at $2 \mathrm{~h}$ with a 77\% reduction (Supplementary Fig. S2).

Phage LM99 demonstrated significant antimicrobial activity against E. faecalis 2133201 (Fig. 3b). This phage was able to sig- nificantly reduce $E$. faecalis 2133201 density by $99 \%$ at 2,6 and $24 \mathrm{~h}$ (Fig. 3b). The other E. faecalis isolates were not significantly affected by the presence of phage LM99 (Supplementary Fig. S2).

Regarding E. coli, phage JB75 demonstrated a significant effect on E. coli 2129975 planktonic cells (Fig. 3c). The highest reduction was achieved at $6 \mathrm{~h}$ of incubation with a 96\% reduction. At $2 \mathrm{~h}$ and 

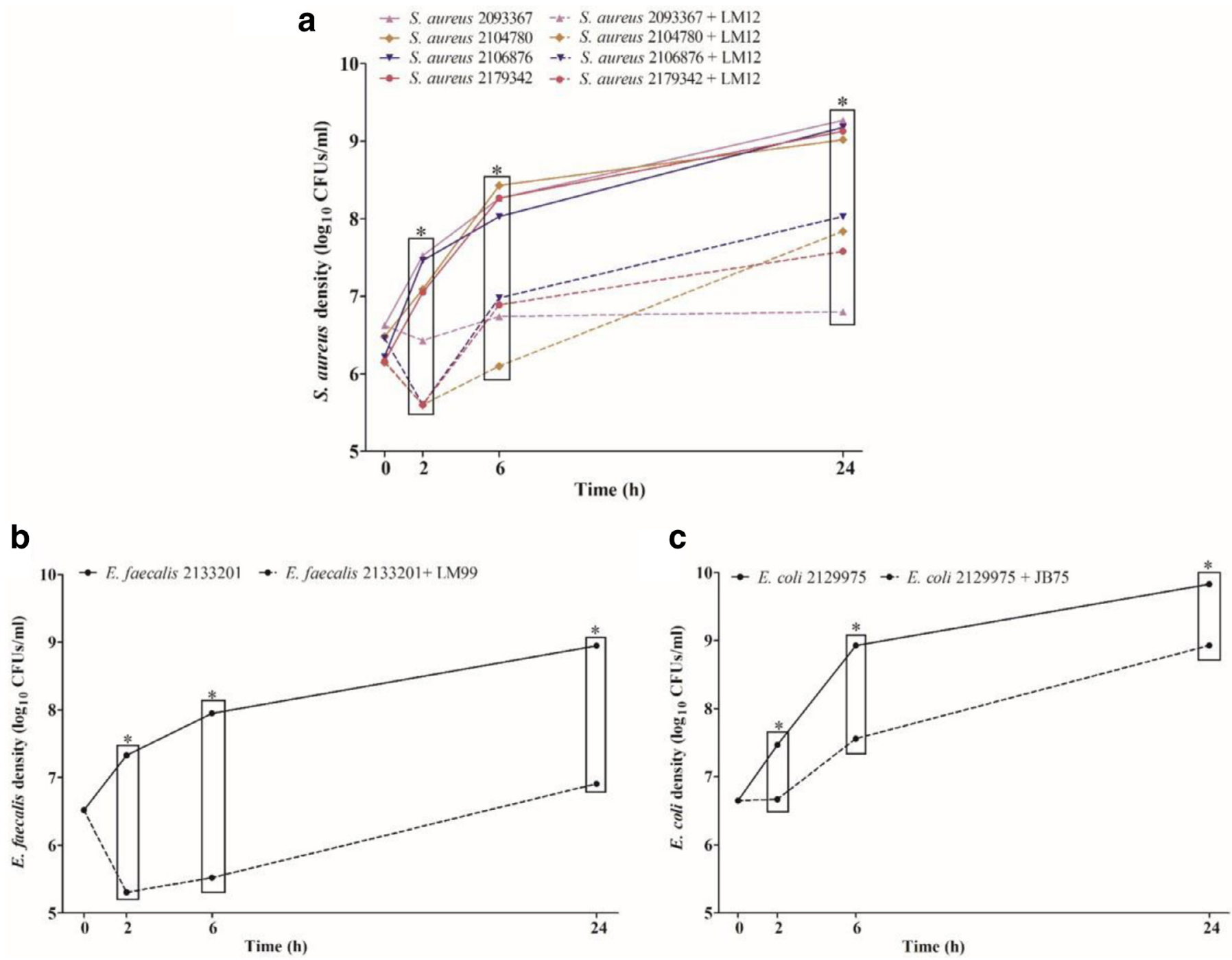

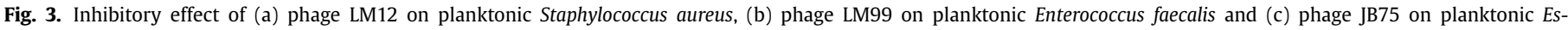

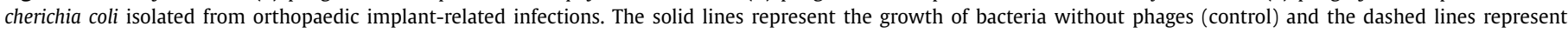

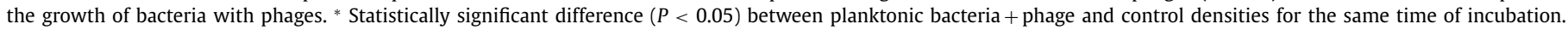

$24 \mathrm{~h}$, the phage was able to reduce the bacterium by $84 \%$ and $87 \%$, respectively (Fig. 3c).

\section{Discussion}

Orthopaedic implant-related infections remain one of the major complications of orthopaedic clinical activity, causing a significant impact on patients and healthcare systems. In addition, the presence of MDR bacteria, namely MRSA and VRE, increases concern about these type of infections owing to limited therapeutic options $[2,3]$. The pathogenicity of these infections is even greater when the isolates possess mobile genetic elements (mecA in S. aureus, Tn916/Tn1545 in E. faecalis and intl1 in E. coli) and virulence elements (such as icaB, hlb and cna in S. aureus, agg, gelE, cylM, cylL and $f_{s r}$ in E. faecalis and fimA in E. coli) [6,27-30]. These mobile genetic elements can mediate the transfer and integration of resistance and virulence determinants into new host DNA [30]. The virulence elements can contribute to bacterial binding to host matrix proteins and consequently bacterial adhesion to implants [6,27]. As observed by MLST and eBURST V3 analysis, both the phenotypic and genotypic profile obtained are in accordance with the findings of other authors [2,3,6,27,31], showing that these pathogens belong to lineages frequently involved in pandemic nosocomial infections [27,31]. Besides, intraspecies diversity between isolates was observed with different resistance and virulence patterns, increasing concern about the diagnosis and treatment of infections caused by these type of bacteria [6,31].

Considering the mentioned pathogenicity of bacterial strains and the poor bioavailability of antibiotics in bone tissue [32], there is an urgent need to develop novel therapeutic approaches to combat isolates involved in orthopaedic implant-associated infections. Phage therapy has long been shown to be a promising antibacterial strategy, mainly due to its high specificity and effectiveness in killing targeted pathogenic bacteria [7,10]. In the present study, three phages, namely LM12 (previously isolated), LM99 (isolated in this study) and JB75 (isolated in this study), were characterised in order to control bacterial pathogens previously isolated from orthopaedic implant-associated infections. The three phages presented outstanding characteristics such as broad bactericidal spectrum against the target pathogenic bacterium, short latent periods, large burst sizes and high stability to several environmental conditions. Furthermore, it is important to highlight the specificity of the phages used in this study. The characterised phages belong to the Caudovirales order, which are doublestranded DNA viruses, having as a major advantage their inherent combined bacteriostatic and bacteriolytic mode of action [33]. By 
comparative genomics analysis, phage LM99 is not inserted in any genus, consequently a new genus should be created comprising LM99 and its closest relative. The homologies detected for phage JB75 and the more identic phages suggest the assignment of this phage to the T4 virus genus, which is one of the most well studied Enterobacteriaceae strictly lytic phages [34]. Furthermore, the phages are virulent and do not encode any genes associated with lysogeny. Genes homologous to a putative toxin gene (gp17 - haemolysin) and to a metallo- $\beta$-lactamase gene were detected in the genome of phage LM99. However, both genes are not homologous to genes found in bacteria, therefore their true function needs to be determined. All of these characteristics associated with the high efficiency of phages LM12, LM99 and JB75 in infecting bacteria, including MRSA and VRE isolated from orthopaedic implant-associated infections, make them potential candidates in therapeutic applications. Likewise, the three studied phages were efficient in reducing the number of culturable bacterial cells over time compared with controls. Phage therapy studies with animal models have shown that, under certain circumstances, phages may help in reducing the density of the infecting bacterial population to a level that may allow the host immune response to mount a successful defence and clear the infection [7]. Recent studies have shown the efficacy and accuracy of phage therapy in the treatment of wound infections, diabetic foot ulcers, acute kidney injury, ulcers and chronic otitis caused by clinical pathogens [35-39]. In line with these potential clinical applications and considering the data obtained in the present work, phages LM12, LM99 and JB75 could be suitable to treat orthopaedic implant-associated infections.

\section{Conclusions}

Bacteria isolated from orthopaedic implant-associated infections showed patterns of multidrug resistance, virulence and pathogenicity. Bacteriophage therapy offers a possible alternative to classic antibiotic treatment to reduce bacterial colonisation. The studied phages were efficient in controlling clinical bacteria, suggesting that phage therapy could be suitable to treat pathogenic bacteria involved in orthopaedic implant-associated infections.

\section{Declaration of Competing Interest}

None declared.

\section{Acknowledgment}

The authors would like to acknowledge Dr Rui Fernandes (HEMS/i3S, Porto, Portugal) for help with electron microscopy analysis, and Margarida Sousa, PhD [Universidade de Trás-os-Montes e Alto Douro (UTAD), Vila Real, Portugal)] for support with the experiments in the Medical Microbiology Laboratory of UTAD.

\section{Funding}

This work was supported by the European Regional Development Fund (FEDER) through the COMPETE 2020 - Operacional Programme for Competitiveness and Internationalisation (POCI), Portugal 2020; by Portuguese funds through FCT/MCTES in the framework of the project 'Institute for Research and Innovation in Health Sciences' [POCI-01-0145-FEDER-007274]; by Project Biotherapies [NORTE-01-0145-FEDER-000012]; and by a PhD grant to JB [SFRH/BD/102148/2014].

\section{Ethical approval}

Not required.

\section{Supplementary materials}

Supplementary material associated with this article can be found, in the online version, at doi:10.1016/j.ijantimicag.2019.06. 007.

\section{References}

[1] Arciola CR, Campoccia D, Montanaro L. Implant infections: adhesion, biofilm formation and immune evasion. Nat Rev Microbiol 2018;16:397-409.

[2] Tande AJ, Patel R. Prosthetic joint infection. Clin Microbiol Rev 2014;27:302-45.

[3] Cremet L, Corvec S, Bemer P, Bret L, Lebrun C, Lesimple B, et al. Orthopaedic-implant infections by Escherichia coli: molecular and phenotypic analysis of the causative strains. J Infect 2012;64:169-75.

[4] Landraud L, Jaureguy F, Frapy E, Guigon G, Gouriou S, Carbonnelle E, et al. Severity of Escherichia coli bacteraemia is independent of the intrinsic virulence of the strains assessed in a mouse model. Clin Microbiol Infect 2013;19:85-90.

[5] Frank KL, Vergidis P, Brinkman CL, Greenwood Quaintance KE, Barnes AM, Mandrekar JN, et al. Evaluation of the Enterococcus faecalis biofilm-associated virulence factors AhrC and Eep in rat foreign body osteomyelitis and in vitro biofilm-associated antimicrobial resistance. PLoS One 2015;10:e0130187.

[6] Montanaro L, Arciola CR, Baldassarri L, Borsetti E. Presence and expression of collagen adhesin gene (cna) and slime production in Staphylococcus aureus strains from orthopaedic prosthesis infections. Biomaterials 1999;20:1945-9.

[7] Cheng M, Liang J, Zhang Y, Hu L, Gong P, Cai R, et al. The bacteriophage EF-P29 efficiently protects against lethal vancomycin-resistant Enterococcus faecalis and alleviates gut microbiota imbalance in a murine bacteremia model. Front Microbiol 2017;8:837.

[8] World Health Organization (WHO). WHO publishes list of bacteria for which new antibiotics are urgently needed. Geneva, Switzerland: WHO; 2017 https://www.who.int/news-room/detail/27-02-2017-who-publishes-listof-bacteria-for-which-new-antibiotics-are-urgently-needed [Accessed 25 July 2019].

[9] Pires DP, Vilas Boas D, Sillankorva S, Azeredo J. Phage therapy: a step forward in the treatment of Pseudomonas aeruginosa infections. J Virol 2015;89:7449-56.

[10] Oliveira A, Sillankorva S, Quinta R, Henriques A, Sereno R, Azeredo J. Isolation and characterization of bacteriophages for avian pathogenic E. coli strains. J Appl Microbiol 2009;106:1919-27.

[11] Capparelli R, Parlato M, Borriello G, Salvatore P, Iannelli D. Experimental phage therapy against Staphylococcus aureus in mice. Antimicrob Agents Chemother 2007;51:2765-73.

[12] European Committee on Antimicrobial Susceptibility Testing. Breakpoint tables for interpretation of MICs and zone diameters; 2015. Version 5.0 http://www. eucast.org [Accessed 26 July 2019].

[13] Clinical and Laboratory Standards Institute (CLSI). Performance standards for antimicrobial susceptibility testing; twenty-fifth informational supplement, Wayne, PA: CLSI; 2015. CLSI document M100-S25.

[14] Sousa M, Silva N, Igrejas G, Silva F, Sargo R, Alegria N, et al. Antimicrobial resistance determinants in Staphylococcus spp. recovered from birds of prey in Portugal. Vet Microbiol 2014;171:436-40.

[15] Goncalves A, Igrejas G, Radhouani H, Correia S, Pacheco R, Santos T, et al. Antimicrobial resistance in faecal enterococci and Escherichia coli isolates recovered from Iberian wolf. Lett Appl Microbiol 2013;56:268-74.

[16] Lozano C, Porres-Osante N, Crettaz J, Rojo-Bezares B, Benito D, Olarte I, et al. Changes in genetic lineages, resistance, and virulence in clinical methicillin-resistant Staphylococcus aureus in a Spanish hospital. J Infect Chemother 2013;19:233-42

[17] Radhouani H, Pinto L, Coelho C, Sargo R, Araujo C, Lopez M, et al. MLST and a genetic study of antibiotic resistance and virulence factors in vanA-containing Enterococcus from buzzards (Buteo buteo). Lett Appl Microbiol 2010;50:537-41.

[18] Ruiz J, Simon K, Horcajada JP, Velasco M, Barranco M, Roig G, et al. Differences in virulence factors among clinical isolates of Escherichia coli causing cystitis and pyelonephritis in women and prostatitis in men. J Clin Microbiol 2002;40:4445-9.

[19] Melo LDR, Brandao A, Akturk E, Santos SB, Azeredo J. Characterization of a new Staphylococcus aureus kayvirus harboring a lysin active against biofilms. Viruses 2018;10 pii: E182. doi:10.3390/v10040182.

[20] van Belkum A, Goessens W, van der Schee C, Lemmens-den Toom N, Vos MC Cornelissen J, et al. Rapid emergence of ciprofloxacin-resistant Enterobacteriaceae containing multiple gentamicin resistance-associated integrons in a Dutch hospital. Emerg Infect Dis 2001;7:862-71.

[21] Sillankorva S, Neubauer P, Azeredo J. Isolation and characterization of a T7-like lytic phage for Pseudomonas fluorescens. BMC Biotechnol 2008;8:80.

[22] Kvachadze L, Balarjishvili N, Meskhi T, Tevdoradze E, Skhirtladze N, Pataridze T, et al. Evaluation of lytic activity of staphylococcal bacteriophage Sb-1 against freshly isolated clinical pathogens. Microb Biotechnol 2011;4:643-50.

[23] Li B, Webster TJ. Bacteria antibiotic resistance: new challenges and opportunities for implant-associated orthopedic infections. J Orthop Res 2018;36:22-32.

[24] Melo LD, Sillankorva S, Ackermann HW, Kropinski AM, Azeredo J, Cerca N. Isolation and characterization of a new Staphylococcus epidermidis broad-spectrum bacteriophage. J Gen Virol 2014;95:506-15. 
[25] Magiorakos AP, Srinivasan A, Carey RB, Carmeli Y, Falagas ME, Giske CG, et al. Multidrug-resistant, extensively drug-resistant and pandrug-resistant bacteria: an international expert proposal for interim standard definitions for acquired resistance. Clin Microbiol Infect 2012;18:268-81.

[26] Kropinski AM, Prangishvili D, Lavigne R. Position paper: the creation of a rational scheme for the nomenclature of viruses of bacteria and Archaea. Environ Microbiol 2009;11:2775-7.

[27] Quinones D, Kobayashi N, Nagashima S. Molecular epidemiologic analysis of Enterococcus faecalis isolates in Cuba by multilocus sequence typing. Microb Drug Resist 2009;15:287-93.

[28] Baldassarri L, Creti R, Recchia S, Pataracchia M, Alfarone G, Orefici G, et al. Virulence factors in enterococcal infections of orthopedic devices. Int J Artif Organs 2006;29:402-6.

[29] Montanaro L, Speziale P, Campoccia D, Ravaioli S, Cangini I, Pietrocola G, et al. Scenery of Staphylococcus implant infections in orthopedics. Future Microbiol 2011;6:1329-49.

[30] Malachowa N, DeLeo FR. Mobile genetic elements of Staphylococcus aureus. Cell Mol Life Sci 2010;67:3057-71.

[31] Ruiz-Garbajosa P, Bonten MJ, Robinson DA, Top J, Nallapareddy SR, Torres C, et al. Multilocus sequence typing scheme for Enterococcus faecalis reveals hospital-adapted genetic complexes in a background of high rates of recombination. J Clin Microbiol 2006;44:2220-8.

[32] Olson ME, Horswill AR. Staphylococcus aureus osteomyelitis: bad to the bone. Cell Host Microbe 2013;13:629-31.
[33] Roach DR, Leung CY, Henry M, Morello E, Singh D, Di Santo JP, et al. Synergy between the host immune system and bacteriophage is essential for successful phage therapy against an acute respiratory pathogen. Cell Host Microbe 2017;22:38-47 e4.

[34] Miller ES, Kutter E, Mosig G, Arisaka F, Kunisawa T, Ruger W. Bacteriophage T4 genome. Microbiol Mol Biol Rev 2003;67:86-156 table of contents.

[35] Watanabe R, Matsumoto T, Sano G, Ishii Y, Tateda K, Sumiyama Y, et al. Efficacy of bacteriophage therapy against gut-derived sepsis caused by Pseudomonas aeruginosa in mice. Antimicrob Agents Chemother 2007;51:446-52.

[36] Biswas B, Adhya S, Washart P, Paul B, Trostel AN, Powell B, et al. Bacteriophage therapy rescues mice bacteremic from a clinical isolate of vancomycin-resistant Enterococcus faecium. Infect Immun 2002;70:204-10.

[37] Wang J, Hu B, Xu M, Yan Q, Liu S, Zhu X, et al. Therapeutic effectiveness of bacteriophages in the rescue of mice with extended spectrum $\beta$-lactamase-producing Escherichia coli bacteremia. Int $\mathrm{J}$ Mol Med 2006; 17:347-55.

[38] Wills QF, Kerrigan C, Soothill JS. Experimental bacteriophage protection against Staphylococcus aureus abscesses in a rabbit model. Antimicrob Agents Chemother 2005;49:1220-1.

[39] Wright A, Hawkins CH, Anggard EE, Harper DR. A controlled clinical trial of a therapeutic bacteriophage preparation in chronic otitis due to antibiotic-resistant Pseudomonas aeruginosa; a preliminary report of efficacy. Clin Otolaryngol 2009;34:349-57. 